\title{
3D Cell Culture Model for Prostate Cancer Cells to Mimic Inflammatory Microenvironment ${ }^{\dagger}$
}

\author{
Gizem Gulevin Takir ${ }^{1}$, Bilge Debelec-Butuner ${ }^{2}$ and Kemal Sami Korkmaz 3,* \\ 1 Graduate School of Natural and Applied Science, Department of Biotechnology, Ege University, \\ 35040 Izmir, Turkey; g.takir@gmail.com \\ 2 Department of Pharmaceutical Biotechnology, Faculty of Pharmacy, Ege University, 35040 Izmir, Turkey; \\ bilge.debelec@ege.edu.tr \\ 3 Bioengineering Department, Faculty of Engineering, Ege University, 35040 Izmir, Turkey \\ * Correspondence: ks_korkmaz@yahoo.com; Tel.: +90-232-311-5808 \\ + Presented at the 2nd International Cell Death Research Congress, Izmir, Turkey, 1-4 November 2018.
}

Published: 6 December 2018

\begin{abstract}
The studies on the relationship between inflammation and cancer progression have been mostly carried out with monolayer cell cultures in vitro, which can be insufficient to mimic tumor tissue. Here, we established a three-dimensional (3D) cell culture model of inflammatory microenvironment for prostate cancer cells to better evaluate the role of inflammation in prostate carcinogenesis. Formation of the cell spheroids has been achieved for LNCaP, Du145, LNCaP-104r2 prostate cancer cell lines but not for RWPE1 normal prostate epithelial cell and PC3 by using 3D Petri Dish ${ }^{\circledR}$. We also showed that cells in inflammatory conditioned media might have a different response based on the culturing method. Overall, we are suggesting that 3D cell culture model can be a useful tool to study molecular alterations on proliferation and migration/invasion of tumor cells related to inflammation.
\end{abstract}

Keywords: 3D culture; prostate cancer; inflammation

\section{Introduction}

Progression of prostate cancer mainly depends on the advanced age and genetic predisposition. Lately, studies have also focused on the effect of inflammatory microenvironment on prostate cancer progression, showing especially chronic inflammation, which is responsible for cancer initiation at $20 \%$ of adult cancers [1].

Unfortunately, the studies of the relation between inflammation and prostate cancer have been mainly restricted to rodent models because of the insufficient complexity of in vitro cultures [2]. Although monolayer cells cultures are occasionally enough to analyze the changes in cell behavior against treatments, the techniques can mislead the results by disrupting cell-cell connections. To overcome these problems, researchers tend to use 3D cultures since they reflect in vivo conditions such as cell-cell interactions, cell polarity, mass transfer and so on more accurately [3].

Our study aims to form spheroids from monolayer prostate cells and to validate the previously established inflammatory microenvironment model on 3D cell culture.

\section{Materials and Methods}

\subsection{The Preparation of Conditioned Media (CM) and Measurement of Included Cytokines}

To prepare CM, U937 monocyte cell line [in RPMI 1640 medium with 10\% FBS (fetal bovine serum) at $37{ }^{\circ} \mathrm{C}$ with $5 \% \mathrm{CO}_{2}$ ] was differentiated to macrophage and induced by phorbol acetate 
(PMA) and lipopolysaccharide (LPS), respectively as described previously [4]. The supernatant prepared from induced macrophages (conditioned medium-CM) was assessed with ELISA assay for TNFa (Invitrogen, Carlsbad, CA, USA), interleukin-6 (IL-6) and interleukin-1beta (IL-1b; Boster Biological Technology Co., Fremont, CA, USA) levels according to the manufacturer's recommendations following its filtration $(0.2 \mathrm{~mm})$. Optimized dose and courses of $\mathrm{CM}$ treatment were applied to the cells to induce the inflammatory response.

\section{2. $2 D$ Cell Culture and Treatments}

RWPE-1, LNCaP, DU145, and PC3 cells were obtained from American Type Culture Collection (ATCC, Manassas, VA, USA). 104R2 was requested from the originator laboratory. Normal prostate epithelial cells, RWPE-1, were cultured in Keratinocyte growth medium supplemented with Bovine pituitary extract and $5 \mathrm{mM}$ EGF. While LNCaP cells were propagated in RPMI 1640 with $10 \%$ FBS, DU145 and PC3 cells were cultured in DMEM-F12 with 5\% FBS adding by L-glutamine (2 mM), penicillin $(100 \mathrm{U} / \mathrm{mL})$, and streptomycin $(100 \mathrm{mg} / \mathrm{mL})$. Complete RPMI 1640 with $10 \%$ CT-FBS was used for $104 \mathrm{R} 2$ cells. All cell lines were maintained in standard cell culture conditions $\left(37^{\circ} \mathrm{C}, 5 \%\right.$ CO2, 95\% humidity).

For induction of NF- $\kappa$ B signaling, cells were fed with CM containing $500 \mathrm{pg} / \mathrm{mL}$ TNF $\alpha$ or 25 $\mathrm{ng} / \mathrm{mL}$ recombinant TNF $\alpha(\mathrm{rTNF} \alpha)$ for $6 \mathrm{~h}$.

\subsection{D Cell Culture and Transfer of Spheroids}

In order to generate equal spheroids, 3D Petri Dishes with 96-well (Sigma-Aldrich, St. Louis, MO, USA) were used according to the manufacturer's recommendation [5]. Following $24 \mathrm{~h}$ incubation of spheroids in 3D Petri Dish at the standard culture conditions, spheroids were transferred to the agarose-coated $60 \mathrm{~mm}$ plates. For the transfer of spheroids, the technique, named liquid pressure, was improved based on washing spheroids from 3D Petri Dish. Briefly, the medium surrounding the molds was discarded, and vertically positioned 3D Petri Dishes in plates were washed with medium or treatment solution at least three times. To enhance the effectiveness of this technique, 3D Petri Dishes were also incubated upside down in the culture for $10 \mathrm{~min}$.

\subsection{Imaging and Western Blot}

To monitor the spheroids, bright field images were captured by inverted microscope Olympus-CX41 (Olympus, Tokyo, Japan). Subsequent to the collection of spheroids, the analysis of proteins was performed by western blotting as described previously [4]. Following proteins were used; NFkB/p65, p-IkB- $\alpha$ (Ser32/36) (Santa Cruz, Inc., Santa Cruz, CA, USA), p-NFkB/p65(S536), IkB- $\alpha$ (Cell Signaling Inc., Danvers, MA, USA). HRP conjugated $\beta$-actin (Sigma-Aldrich, Hamburg, Germany) was used as a loading control. The HRP-anti-mouse and HRP-anti-rabbit secondary antibodies were purchased from Amersham, UK.

\section{Results and Discussion}

\subsection{Formation and Transfer of the Spheroids}

We tested five different prostate cells (RWPE-1, normal prostate epithelial cell line, LNCaP, DU145, and PC3 metastatic prostate cancer cell lines, LNCaP 104R2, LNCaP-derived castration-resistant cell line) to see if they are compatible with spheroid formation in our conditions. $24 \mathrm{~h}$ after the incubation of cells in 3D Petri Dishes, the spheroids were analyzed by phase contrast microscope. We confirmed that all cell lines used in this study assembled in wells properly (Figure 1).

In order to detect whether cells preserve their spherical shapes, we transferred spheroids to 60 $\mathrm{mm}$ dishes following the formation of spheroids in 3D Petri Dishes. $6 \mathrm{~h}$ after the transfer, it was observed that LNCaP, 104R2 and DU145 cells maintained spherical shapes, whereas RWPE-1 and PC3 cells collapsed (Figure 1). 


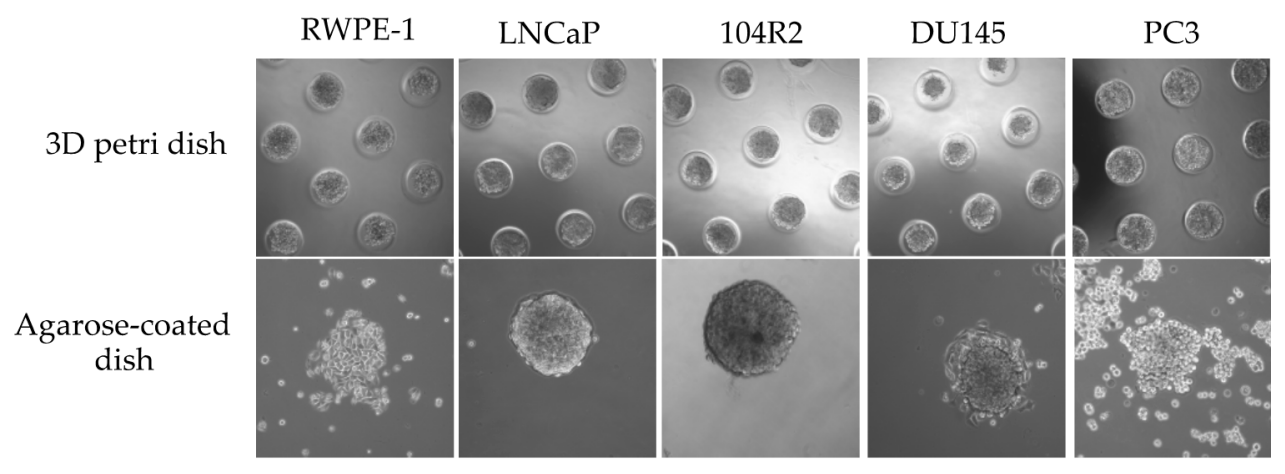

Figure 1. The phase-contrast images of spheroids formed in 3D Petri Dish and post-transferred spheroids in 6-well plates. $24 \mathrm{~h}$ after the incubation, cells uniformly formed in 3D Petri Dish (upper panel). Representative images showed the transfer response for every cell line after $6 \mathrm{~h}$ (bottom panel). Bar: 100 (upper panel) and $150 \mu \mathrm{m}$ (bottom panel).

\subsection{The Effect of CM Treatment Is Alleviated in $3 D$ Compared to $2 D$}

To analyze the effect of inflammatory microenvironment on cancer progression, we treated LNCaP cells with conditioned medium (TNF $\alpha$ concentration is $500 \mathrm{pg} / \mathrm{mL}$ ) for both 3D and 2D cultures. We also applied recombinant TNF $\alpha(25 \mathrm{ng} / \mathrm{mL})$ to $2 \mathrm{D}$ cultures as a positive control of NF- $\kappa B$ activation.

Any morphological changes based on the size of spheroids were not observed in CM-treated cells when compared to the untreated group. In addition, activation of NFkB signaling pathway was validated in 3D culture in LNCaPs (Figure 2). As expected, NFkB/p65 level stayed stable whereas increased p-NFkB/p65(S536), which has been previously shown in 2D culture [4], was observed in the CM-treated group in comparison to the control group 3D culture. On the other hand, increased

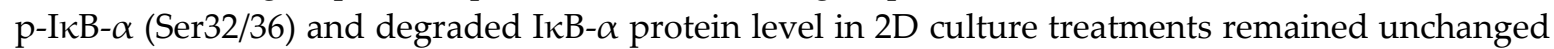
in 3D culture (Figure 2).

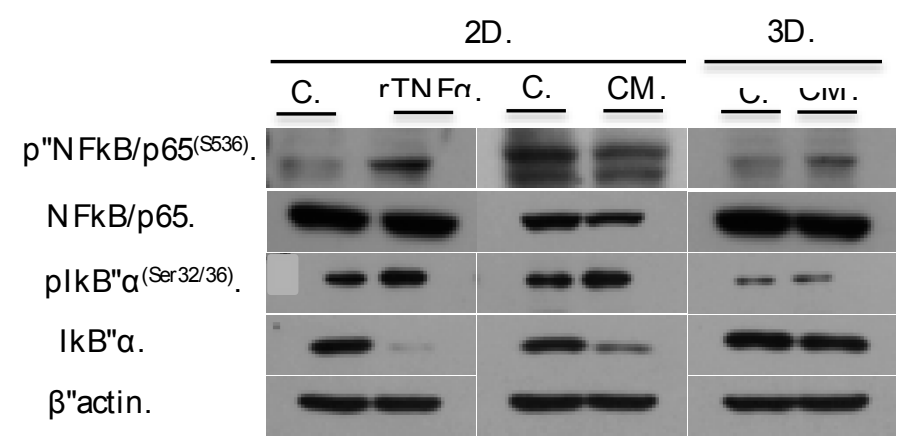

Figure 2. The effect of inflammation on LNCaP cells. Dependent on CM (500 pg/mL) or rTNF- $\alpha$ (25 $\mathrm{ng} / \mathrm{mL}$ ) exposure, the protein level of $\mathrm{p}-\mathrm{NFkB}$ were increased whereas NFkB was protected. The expression level of $\mathrm{I} \kappa \mathrm{B} \alpha$ and $\mathrm{p}-\mathrm{I} \kappa \mathrm{B} \alpha$ remained stable in $3 \mathrm{D}$ cultured cells. The equal loading of lysates was shown by $\beta$-actin. Two independent experiments were performed.

Overall, those results indicated that in vitro inflammatory microenvironment model in 3D cell culture is a useful tool for studies on inflammation-related carcinogenesis, however, appropriate positive controls for comparison to $2 \mathrm{D}$ cell culture and further optimization for different cell lines are still needed for reliable results.

Author Contributions: All authors contributed equally to this work.

Funding: The project was supported by the Turkish Scientific and Technological Research Council (grants TUBITAK-113S044). 
Acknowledgments: We would like to thank A. Sendemir-Urkmez for providing the micro-molds ${ }^{\circledR}$ (Sigma-Aldrich).

Conflicts of Interest: The authors declare no conflict of interest.

\section{References}

1. Marzo, A.M.D.; Platz, E.A.; Sutcliffe, S.; Xu, J.; Grönberg, H.; Drake, C.G.; Nakai, Y.; Isaacs, W.B.; Nelson, W.G. Inflammation in prostate carcinogenesis. Nat. Rev. Cancer 2007, 7, 256-269.

2. Haverkamp, J.; Charbonneau, B.; Ratliff, T.L. Prostate inflammation and its potential impact on prostate cancer: A current review. J. Cell. Biochem. 2008, 103, 1344-1353.

3. Costa, E.C.; Moreira, A.F.; de Melo-Diogo, D.; Gaspar, V.M.; Carvalho, M.P.; Correia, I.J. 3D tumor spheroids: An overview on the tools and techniques used for their analysis. Biotechnol. Adv. 2016, 34, 1427-1441.

4. Debelec-Butuner, B.; Alapinar, C.; Varisli, L.; Erbaykent-Tepedelen, B.; Hamid, S.M.; Gonen-Korkmaz, C.; Korkmaz, K.S. Inflammation-mediated abrogation of androgen signaling: An in vitro model of prostate cell inflammation. Mol. Carcinog. 2014, 53, 85-97.

5. Microsoft Word-Casting, Equilibrating and Seeding the 3D Petri Dish ${ }^{\circledR}$.docx. Available online: http://ftp.microtissues.com/3DP/3dcellculture_protocols/Casting_Equilibrating_and_Seeding_the_3D_Pet ri_Dish.pdf (accessed on 14 October 2018).

(C) 2018 by the authors; licensee MDPI, Basel, Switzerland. This article is an open access article distributed under the terms and conditions of the Creative Commons Attribution (CC BY) license (http://creativecommons.org/licenses/by/4.0/). 